\title{
Torsion in Groups of Integral Triangles
}

\author{
Will Murray \\ Department of Mathematics and Statistics, California State University, Long Beach, USA \\ Email: Will.Murray@csulb.edu
}

Received August 22, 2012; revised October 19, 2012; accepted November 5, 2012

\begin{abstract}
Let $0<\gamma<\pi$ be a fixed pythagorean angle. We study the abelian group $H_{\gamma}$ of primitive integral triangles $(a, b, c)$ for which the angle opposite side $c$ is $\gamma$. Addition in $H_{\gamma}$ is defined by adding the angles $\beta$ opposite side $b$ and modding out by $\pi-\gamma$. The only $H_{\gamma}$ for which the structure is known is $H_{\pi / 2}$, which is free abelian. We prove that for general $\gamma, H_{\gamma}$ has an element of order two iff $2(1-\cos \gamma)$ is a rational square, and it has elements of order three iff the cubic $(2 \cos \gamma) x^{3}-3 x^{2}+1=0$ has a rational solution $0<x<1$. This shows that the set of values of $\gamma$ for which $H_{\gamma}$ has two-torsion is dense in $[0, \pi]$, and similarly for three-torsion. We also show that there is at most one copy of either $\mathbb{Z}_{2}$ or $\mathbb{Z}_{3}$ in $H_{\gamma}$. Finally, we give some examples of higher order torsion elements in $H_{\gamma}$.

Keywords: Abelian Groups; Cubic Equations; Examples; Free Abelian; Geometric Constructions; Group Theory; Integral Triangles; Law of Cosines; Primitive; Pythagorean Angles; Pythagorean Triangles; Pythagorean Triples; Rational Squares, Three-Torsion; Torsion; Torsion-Free; Two-Torsion; Triangle Geometry
\end{abstract}

\section{Introduction}

In [1], Olga Taussky introduces a group operation on the set of primitive pythagorean triples

$$
\left\{(a, b, c): \operatorname{gcd}(a, b, c)=1, a^{2}+b^{2}=c^{2}\right\},
$$

that is, the set of primitive integral triangles $(a, b, c)$ in which the angle $\gamma$ opposite side $c$ is a right angle. Ernest J. Eckert spells out the details of the group operation in [2]:

$$
\begin{aligned}
& (a, b, c)+(A, B, C) \\
& := \begin{cases}(a A-b B, a B+b A, c C) & \text { if } a A-b B>0, \\
(a B+b A, b B-a A, c C) & \text { if } a A-b B \leq 0,\end{cases}
\end{aligned}
$$

where we reduce the answer by a common factor if necessary to obtain a primitive triple. The group has identity $(1,0,1)$; the inverse of $(a, b, c)$ is $(b, a, c)$.

The construction arises from the famous identity on sums of squares:

$$
\left(a^{2}+b^{2}\right)\left(B^{2}+B^{2}\right)=(a A-b B)^{2}+(a B+b A)^{2} .
$$

This identity, of course, corresponds to the rule $|z||w|=|z w|$ for complex numbers, and the group law on pythagorean triples has a similar geometric motivation: we identify the triangle $(a, b, c)$ with the complex number $a+b i$, and the group operation is then just complex multiplication.
The argument of $a+b i$ is the angle $\beta$ opposite side $b$ of the triangle. Since we add the arguments of complex numbers when we multiply the numbers, we can interpret the group operation as addition of the angles $\beta_{1}$ and $\beta_{2}$ to find a primitive triple whose corresponding angle is $\beta_{1}+\beta_{2}$. A problem arises when $\beta_{1}+\beta_{2} \geq \frac{\pi}{2}$, i.e. when $a A-b B<0$, since this no longer corresponds to a pythagorean triple with positive coordinates. To fix this, we mod out the angle by $\pi / 2$; this is why we have the two cases in the definition of the group operation above.

This group has been the subject of much interest over the years. In [3], Jean Mariani studies the group of homogeneous linear transformations with integral coefficients that preserve the triangles above as a set. In [4], Barbara Margolius uses a sequence of pythagorean triples derived from the group law above to prove that Plouffe's constant $\frac{\arctan \frac{1}{2}}{\pi}$ is transcendental, a special case of Hilbert's seventh problem.

Eckert's main result in [2] is that this group is free abelian, that is, isomorphic to a direct sum of copies of the integers. The sum is indexed by the triangles $(a, b, p)$, where $a>b$ and $p$ is prime with $p \equiv 1(\bmod 4)$. In particular, the group is torsion-free, that is, there do not 
exist any nontrivial elements $x \neq 0$ with $n x=0$ for $n>0$.

In [5], Eckert and Preben Dahl Vestergaard generalize the construction above to the case when $\gamma$ is no longer a right angle. We define an angle $0<\gamma<\pi$ to be $p y-$ thagorean if $\gamma$ or $\pi-\gamma$ is an angle in a pythagorean triangle, or, equivalently, if $\sin \gamma$ and $\cos \gamma$ are both rational. For a fixed pythagorean angle $\gamma$, we define $H_{\gamma}$ to be the set of primitive integral triangles $(a, b, c)$ for which the angle opposite side $c$ is $\gamma$. We can add two triangles by adding their values for $\beta$, modulo $\pi-\gamma$ :

$$
\begin{aligned}
& (a, b, c)+(A, B, C) \\
& :=\left\{\begin{array}{l}
(a A-b B, a B+b A-2 b B \cos \gamma, c C) \\
(a B+b A-2 a A \cos \gamma, b B-a A, c C)
\end{array}\right.
\end{aligned}
$$

We use the first formula above in the case when $a A-b B>0$, which corresponds to $\beta_{1}+\beta_{2}<\pi-\gamma$, and the second when $a A-b B \leq 0$, which corresponds to $\beta_{1}+\beta_{2} \geq \pi-\gamma$. As before, we stipulate that we will scale the answer by a common factor if necessary to obtain a primitive integral triangle. Eckert and Vestergaard prove that this construction again gives us a group, still with identity $(1,0,1)$ and with the inverse of $(a, b, c)$ being $(b, a, c)$. Note that the original group considered in [2] is just the special case $H_{\pi / 2}$, whose structure we know completely.

Eckert and Vestergaard ask a number of interesting questions about the structure of $H_{\gamma}$ in general. They mention that when $\gamma \neq \frac{\pi}{2}, H_{\gamma}$ can have nontrivial torsion elements, that is, elements $x \neq 0$ such that $n x=0$ for some $n>0$, but they do not give details. In this paper, we pursue the study of torsion elements in $H_{\gamma}$. Our main results give necessary and sufficient conditions on $\gamma$ for $H_{\gamma}$ to have two-torsion and threetorsion. From these theorems, it will be easy to see that the set of values of $\gamma$ for which $H_{\gamma}$ has two-torsion is dense in the interval $0<\gamma<\pi$, and similarly for threetorsion. We will also show that there is at most one copy of either $\mathbb{Z}_{2}$ or $\mathbb{Z}_{3}$ in $H_{\gamma}$. Finally, we will give some examples of higher-order torsion elements in $H_{\gamma}$.

\section{Two-Torsion}

Our results on two-torsion and three-torsion are similar in flavor, but the theorem on two-torsion is simpler and significantly easier to prove, so we will start there.

Theorem 1. $H_{\gamma}$ has a point of order two iff $2(1-\cos \gamma)$ is a rational square. When this is true, the point of order two is unique.

Proof of Theorem 1. Since the inverse of $(a, b, c)$ is $(b, a, c)$, a point has order two iff it has the form $(a, a, c)$.
Suppose first that $(a, a, c) \in H_{\gamma}$ is a point of order two. We invoke the Law of Cosines:

$$
\begin{gathered}
c^{2}=a^{2}+b^{2}-2 a a \cos \gamma \\
\frac{c^{2}}{a^{2}}=2(1-\cos \gamma)
\end{gathered}
$$

So $2(1-\cos \gamma)$ is a rational square, as desired.

Conversely, suppose that $2(1-\cos \gamma)=\frac{c^{2}}{a^{2}}$, where $a$, $c \in \mathbb{Z}$ are relatively prime. Note that

$0<2(1-\cos \gamma)<4$, so $0<c<2 a$, so $(a, a, c)$ is a triangle, and the computation above shows that by the Law of Cosines, the angle opposite side $c$ is $\gamma$. Hence $(a, a, c) \in H_{\gamma}$ is a point of order two.

To show that only one point of order two can exist, we note that any two triangles $(a, a, c)$ and $\left(a^{\prime}, a^{\prime}, c^{\prime}\right)$ in $H_{\gamma}$ would have to be scalar multiples of each other, and would therefore be represented by a single primitive integral triangle.

We illustrate Theorem 1 with some quick examples:

1) For $\gamma=\pi / 2$, we have $2(1-\cos \gamma)=2$, and as shown in [2], has no point of order two.

2) For $\gamma=\pi / 3$, we have $2(1-\cos \gamma)=1$, and $H_{\pi / 3}$ contains the point $(1,1,1)$ of order two.

3) For $\gamma=\arccos \left(-\frac{1}{8}\right)$, we have $2(1-\cos \gamma)=\frac{9}{4}$, and $H_{\gamma}$ contains the point $(2,2,3)$ of order two.

Corollary 2. The set of $\gamma$ for which $H_{\gamma}$ contains a point of order two is dense in $[0, \pi]$.

Proof. This follows from the fact that $2(1-\cos \gamma)$ is a continuous function from $[0, \pi]$ onto $[0,4]$ and the rational squares are dense in $[0,4]$

\section{Three-Torsion}

Our result for three-torsion follows the same general spirit as that for two-torsion, but is significantly harder. We first prove the easy result that $H_{\gamma}$ contains at most one copy of $\mathbb{Z}_{3}$; this proof will help set the stage for the proof of our main theorem.

Theorem 3. $H_{\gamma}$ contains at most two points of order three.

Proof of Theorem 3: Suppose $(a, b, c) \in H_{\gamma}$ has order three. Note that its inverse $(b, a, c)$ also has order three, so we may assume without loss of generality that $a>b$. (If $a=b$, then as we saw above, $(a, b, c)$ has order two, so it cannot have order three). Let $\alpha$ and $\beta$ be the angles opposite sides $a$ and $b$ respectively. Since $a>b$, we have $\beta<\alpha$, so $2 \beta<\pi-\gamma$. Thus, when we compute $2(a, b, c)$, we are adding $\beta$ to itself, and there is no modding out by $\pi-\gamma$. The resulting triangle is $2(a, b, c)=(b, a, c)$, which has angle $\alpha$ opposite side $a$, so $2 \beta=\alpha$. But since $\gamma$ is fixed, the equation $2 \beta=\alpha$ 
completely determines the three angles of the triangle, so there is only one primitive triple with these angles. (The other point of order three, of course, is the inverse $(b, a, c))$.

We will continue to use some of the ideas above in the proof of our main theorem on three-torsion. bic

Theorem 4. $H_{\gamma}$ has a point of order three iff the cu-

$$
(2 \cos \gamma) x^{3}-3 x^{2}+1=0
$$

has a rational solution $0<x<1$.

The cubic criterion gives easy answers to two questions: First, given a specified $\gamma$, we can ask whether $H_{\gamma}$ has three-torsion. Assuming that $\gamma$ is pythagorean, we can use the Rational Root Theorem to check quickly whether the cubic above has a rational solution between 0 and 1 . If it does, the proof below will give a constructive algorithm to produce a triangle $(a, b, c)$ of order three.

Second, if we don't have a specific $\gamma$ in mind, we can use the cubic criterion to find values of $\gamma$ for which $H_{\gamma}$ has three-torsion. Indeed, we can solve the equation above for $\cos \gamma$ :

$$
\cos \gamma=\frac{3 x^{2}-1}{2 x^{3}}
$$

The rational function on the right takes values less than -1 for $0<x<1 / 2$, and it maps the interval $1 / 2 \leq x \leq$ 1 continuously onto the range $[-1,1]$. Therefore, to find angles whose groups have three-torsion, we can take any rational $1 / 2 \leq x \leq 1$ and then find the corresponding $\gamma$.

Proof of Theorem 4. First, suppose that $(a, b, c)$ has order three. As in the proof of Theorem 3 above, we may assume that $a>b$ and that when we compute $2(a, b, c)$, there is no modding out by $\pi-\gamma$. So we use the first set of equations for the group operation:

$$
2(a, b, c)=\left(a^{2}-b^{2}, 2 a b-2 b^{2} \cos \gamma, c^{2}\right) \sim(b, a, c)
$$

The common factor between the two triangles on the right is $c$, giving us the following "scaling equations":

$$
\begin{aligned}
& b c=a^{2}-b^{2} \\
& a c=2 a b-2 b^{2} \cos \gamma=2 b(a-b \cos \gamma)
\end{aligned}
$$

Solve the second scaling equation for $c$ :

$$
c=2 b\left(1-\frac{b}{a} \cos \gamma\right)
$$

Plug this into the Law of Cosines:

$$
a^{2}+b^{2}-2 a b \cos \gamma=4 b^{2}\left(1-2 \frac{b}{a} \cos \gamma+\frac{b^{2}}{a^{2}} \cos ^{2} \gamma\right)
$$

Multiply by $a^{2}$ :

$$
a^{4}+a^{2} b^{2}-2 a^{3} b \cos \gamma=4 a^{2} b^{2}-8 a b^{3} \cos \gamma-4 b^{4} \cos ^{2} \gamma
$$

\section{Rearrange:}

$$
a^{4}-3 a^{2} b^{2}-2 a^{3} b \cos \gamma+8 a b^{3} \cos \gamma-4 b^{4} \cos ^{2} \gamma=0
$$

Factor:

$$
\left[(2 \cos \gamma) b^{3}-3 a b^{2}+a^{3}\right](a-2 b \cos \gamma)=0
$$

We claim that $a-2 b \cos \gamma \neq 0$. To prove this claim, suppose that $a-2 b \cos \gamma=0$; then $b \cos \gamma=\frac{a}{2}$. Plugging this into the second scaling equation, we get $a c=a b$, so $b=c$. Plugging this into the first scaling equation, we get $a^{2}=2 b^{2}$, contradicting the fact that $a$ and $b$ are both integers. This proves our claim, so we may cancel the factor of $a-2 b \cos \gamma$ :

$$
(2 \cos \gamma) b^{3}-3 a b^{2}+a^{3}=0
$$

Divide by $a^{3}$ :

$$
(2 \cos \gamma) \frac{b^{3}}{a^{3}}-3 \frac{b^{2}}{a^{2}}+1=0
$$

Set $x:=\frac{b}{a}$, which is a rational number with $0<x<1$, and we have

$$
(2 \cos \gamma) x^{3}-3 x^{2}+1=0,
$$

as desired.

Conversely, suppose we have a rational solution $0<x$ $<1$ to

$$
(2 \cos \gamma) x^{3}-3 x^{2}+1=0,
$$

Define $a>b$ to be positive relatively prime integers such that $x=\frac{b}{a}$, and define $c$ to satisfy $a c=2 b(a-b \cos \gamma)$. (Note that $c>0)$. Scale $a, b$, and $c$ by a common factor, if necessary, to be relatively prime integers; note that this scaling will preserve both $x=\frac{b}{a}$ and $a c=2 b(a-b \cos \gamma)$.

By reversing the equations from the forward direction of the proof, we can work back to

$$
(2 \cos \gamma) b^{3}-3 a b^{2}+a^{3}=0 .
$$

We can solve this for $b \cos \gamma$ and then build up to $2 b(a-b \cos \gamma)$ as follows:

$$
\begin{aligned}
& b \cos \gamma=\frac{3 a b^{2}-a^{3}}{2 b^{2}} \\
& a-b \cos \gamma=\frac{2 a b^{2}-3 a b^{2}+a^{3}}{2 b^{2}}
\end{aligned}
$$




$$
\begin{aligned}
& a-b \cos \gamma=\frac{a^{3}-a b^{2}}{2 b^{2}} \\
& 2 b(a-b \cos \gamma)=\frac{a^{3}-a b^{2}}{b}
\end{aligned}
$$

Substitute:

$$
a c=\frac{a^{3}-a b^{2}}{b}
$$

Simplify:

$$
b c=a^{2}-b^{2}
$$

Now we check that $(a, b, c)$ is a triangle. First, we already saw that $a>b$, so $b<a+c$. Second, to see that $a<b+c$, we can reorganize the equation immediately above:

$$
\begin{gathered}
b c+b^{2}=a^{2}>a b \\
b(b+c)>a b \\
b+c>a
\end{gathered}
$$

Third, we can continue to reverse the equations from the forward direction of the proof until we get back to

$$
\begin{aligned}
& a^{2}+b^{2}-2 a b \cos \gamma \\
& =4 b^{2}\left(1-2 \frac{b}{a} \cos \gamma+\frac{b^{2}}{a^{2}} \cos ^{2} \gamma\right)=c^{2},
\end{aligned}
$$

which gives us $c<a+b$. It also shows by the Law of Cosines that the triangle $(a, b, c)$, has angle $\gamma$ opposite side $c$, so $(a, b, c) \in H_{\gamma}$.

Finally, we have already established the scaling equations

$$
\begin{gathered}
b c=a^{2}-b^{2} \\
a c=2 b(a-b \cos \gamma)
\end{gathered}
$$

which show that

$$
\begin{aligned}
2(a, b, c) & =\left(a^{2}-b^{2}, 2 a b-2 b^{2} \cos \gamma, c^{2}\right) \sim(b, a, c) \\
& =-(a, b, c),
\end{aligned}
$$

so $(a, b, c)$ has order three, as desired.

Here are some sample applications of Theorem 4:

1) Let $\gamma=\pi / 2$. Then the cubic degenerates to $-3 x^{2}+1=0$, which has no rational solutions at all, and $H_{\pi / 2}$ has no point of order three.

2) Let $\gamma=\arccos \frac{9}{16}$. Then the cubic becomes $\frac{9}{8} x^{3}-3 x^{2}+1=0$, which has the rational solution $x=\frac{2}{3}$. Following this through the proof above leads to the triangle $(6,4,5) \in H_{\gamma}$, which has order three.
3) Let $\gamma=\arccos \left(-\frac{7}{128}\right)$. Then the cubic becomes $-\frac{7}{64} x^{3}-3 x^{2}+1=0$, which has the rational solution $x=\frac{4}{7}$. This leads to the triangle $(28,16,33) \in \mathcal{H}_{\gamma}$, which has order three.

Although it is not quite so obvious, we have the same corollary as for two-torsion:

Corollary 5. The set of $\gamma$ for which $H_{\gamma}$ contains a point of order three is dense in $[0, \pi]$.

Proof. The rationals are dense in the interval $1 / 2 \leq x \leq$ 1 , which, as noted above, is mapped continuously by

$\frac{3 x^{2}-1}{2 x^{3}}$ onto $[-1,1]$ and in turn by arceos onto $0 \leq y \leq$ $\pi$. Hence the set of $\gamma$ corresponding to a rational $x$ is dense in $[0, \pi]$.

\section{Examples of Higher Order Torsion}

We close with some examples of higher order torsion points in $H_{\gamma}$ :

1) Let $\gamma=\arccos \frac{31}{32}$. Then the triangle $(10,8,3)$ has order four in $H_{\gamma}$.

2) Let $\gamma=\arccos \frac{475}{486}$. Then the triangle $(105,81,31)$ has order five in $H_{\gamma}$.

3) Let $\gamma=\arccos \frac{8143}{8192}$. Then the triangle $(1220,1024$, 231) has order six in $H_{\gamma}$.

I found these essentially by a brute force search on a computer, finding multiples of integral triangles and seeing which ones have finite order. As patterns start to emerge for smaller values, we can narrow and speed up the search for larger values by restricting to those triangles that satisfy similar patterns. In particular, there is a clear pattern in the values of $b$ here: $2^{3}, 3^{4}, 4^{5}, \cdots$. From computer-generated evidence, the pattern on the exponents seems to be genuine, but the pattern on the bases is a red herring: other examples of points of order six are $(5555,3125,3024)$ for $\gamma=\arccos \frac{14167}{15625},(17214,7776$, $12155)$ for $\gamma=\arccos \frac{72863}{93312}$, and $(42833,16807,34320)$ for $\gamma=\arccos \frac{76751}{117649}$. The consistent pattern seems to be that a point of order $n$ must have the form $\left(a, k^{n-1}, c\right)$ (or, of course, $\left(k^{n-1}, b, c\right)$ ), but I do not have a proof of this. I invite you to prove this or find a counterexample. 


\section{Conclusions and Further Questions}

We have seen that although $H_{\pi / 2}$ is torsion-free, there are many values $\gamma \in(0, \pi)$ for which $H_{\gamma}$ has torsion points of various orders. In fact, the set of $\gamma$ for which $H_{\gamma}$ has two-torsion (respectively, three-torsion) is dense in $[0, \pi]$, and we can easily characterize all such $\gamma$ in terms of conditions on $\cos \gamma$. We have also seen that in such cases, $H_{\gamma}$ contains a unique copy of $\mathbb{Z}_{2}$ (respectively, $\mathbb{Z}_{2}$ ).

There remain many intriguing open questions on $H_{\gamma}$. Obviously we would like to have a complete determination of the isomorphism type of $H_{\gamma}$ for all $\gamma$, but since that doesn't seem feasible in the near future, there are plenty of more approachable issues:

1) For what values of $n$ do there exist $H_{\gamma}$ with $n$-torsion?

2) For any $n$ for which this is true, how can we characterize the $\gamma$ for which $H_{\gamma}$ has $n$-torsion?

3 ) Is the set of such $\gamma$ dense in $[0, \pi]$ ? (Note that in all the examples of higher order torsion above, we had $\gamma<\pi / 2)$.

4) Is it possible for $H_{\gamma}$ to contain more than one copy of $\mathbb{Z}_{n}$ ?

\section{REFERENCES}

[1] O. Taussky, "Sums of Squares," American Mathematical Monthly, Vol. 77, No. 8, 1970, pp. 805-830. doi: $10.2307 / 2317016$

[2] E. J. Eckert, "The Group of Primitive Pythagorean Triangles," Mathematics Magazine, Vol. 57, No. 1, 1984, pp. 22-27. doi:10.2307/2690291

[3] J. Mariani, "The Group of the Pythagorean Numbers", American Mathematical Monthly, Vol. 69, 1962, pp. 125128. doi: $10.2307 / 2312540$

[4] B. H. Margolius, "Plouffe's Constant is Transcendental." http://www.plouffe.fr/simon/articles/plouffe.pdf

[5] E. J. Eckert and P. D. Vestergaard, "Groups of Integral Triangles,” Fibonacci Quarterly, Vol. 27, No. 5, 1989, pp. 458-464. 